\title{
Note on the conformable boundary value problems: Sturm's theorems and Green's function
}

\author{
F. Martínez ${ }^{a, *}$, I. Martínez ${ }^{a}$, M. K. A. Kaabar ${ }^{b}$ and S. Paredes ${ }^{a}$ \\ ${ }^{a}$ Department of Applied Mathematics and Statistics, \\ Technological University of Cartagena, Spain. \\ Tel: +34968325586; \\ *f.martinez@upct.es \\ ${ }^{b}$ Department of Mathematics and Statistics, \\ Washington State University, Pullman, WA, 99163, USA.
}

Received 12 November 2020; accepted 11 January 2021

Recently, the conformable derivative and its properties have been introduced. In this paper, we propose and prove some new results on conformable Boundary Value Problems. First, we introduce a conformable version of classical Sturm's separation, and comparison theorems. For a conformable Sturm-Liouville problem, Green's function is constructed, and its properties are also studied. In addition, we propose the applicability of the Green's Function in solving conformable inhomogeneous linear differential equations with homogeneous boundary conditions, whose associated homogeneous boundary value problem has only trivial solution. Finally, we prove the generalized Hyers-Ulam stability of the conformable inhomogeneous boundary value problem.

Keywords: Conformable fractional derivative; conformable fractional integral; conformable fractional differential equations; Sturm's theorems; Green's function.

PACS: $02.30 . \mathrm{Hq}$; 02.30.Gp; 02.90. + p

DOI: https://doi.org/10.31349/RevMexFis.67.471

\section{Introduction}

The idea of fractional derivative was first raised by L'Hospital in 1695. Since then, several related new definitions have been proposed. The most common ones are Riemann-Liouville and Caputo definitions. For more information about the most known fractional definitions, we refer to [1,2]. A new definition of fractional derivative and fractional integral has been recently proposed by Khalil et al. in [3]. As a result, several important elements of the mathematical analysis of functions of a real variable have been formulated such as: chain rule, fractional power series expansion and fractional integration by parts formulas, Rolle's Theorem, and Mean Value Theorem [3-5, 40]. The conformable partial derivative of the order $\alpha \in(0.1]$ of the real-valued functions of several variables and conformable gradient vector are also defined. In addition, a conformable version of Clairaut's Theorem for partial derivative is investigated in [6]. In [7], conformable Jacobian matrix is defined, and chain rule for multivariable conformable derivative is proposed. In [8], the conformable version of Euler's Theorem on homogeneous is introduced. Furthermore, in a short time, various research studies have been conducted on the theory and applications of fractional differential equations in the context of this newly introduced fractional derivative [9-18,23,24].

In addition, another new definition of local fractional derivative is introduced by P. M. Guzmán et al. [25], and it is called the non-conformable fractional derivative which is considered as a natural extension of the usual derivative of a function a point. The difference between conformable fractional derivative and non-conformable fractional derivative is that the tangent line angle is conserved in the conformable one, while it is not conserved in the sense of nonconformable one [25] (see also [26,34,36,37] for more new related results about this newly proposed definition of non-conformable fractional derivative). The definition of non-conformable fractional derivative has been investigated and applied in various research studies and applications of physics and natural sciences such as the stability analysis, oscillatory character, and boundedness of fractional Liénard-type systems $[27,28,33]$, analysis of the local fractional Drude model [29], Hermite-Hadamard inequalities [30], fractional Laplace transform [31], fractional logistic growth models [32], oscillatory character of fractional Emden-Fowler equation [35], asymptotic behavior of fractional nonlinear equations [38], and qualitative behavior of nonlinear differential equations [39].

This paper is organized as follows: In Sec. 2, the main concepts of conformable fractional calculus are presented. Section 3, we proved a conformable version of the conformable second-order Sturm-Picone identity. From this result, we establish the conformable Sturm-Liouville comparison and separation theorems. Section 4, for a conformable Sturm-Liouville problem, the Green function is constructed, and its properties are studied. At the end, we prove the generalized Hyers-Ulam stability of conformable inhomogeneous linear differential equations with homogeneous boundary conditions. 


\section{Basic definitions and tools}

Definition 1. Given a function $f:[0 \infty) \rightarrow R$. Then, the conformable fractional derivative of order $\alpha$, [3], is defined by

$$
\left(T_{\alpha} f\right)(t)=\lim _{\varepsilon \rightarrow 0} \frac{f\left(t+\varepsilon t^{1-\alpha}\right)-f(t)}{\varepsilon},
$$

for all $t>0,0<\alpha \leq 1$. If $f$ is $\alpha$-differentiable in some $(0, a), a>0$, and $\lim _{t \rightarrow 0^{+}}\left(T_{\alpha} f\right)(t)$ exists, then it is defined as

$$
\left(T_{\alpha} f\right)(0)=\lim _{t \rightarrow 0^{+}}\left(T_{\alpha} f\right)(t) .
$$

Theorem 1. [3]. If a function $f:[0, \infty) \rightarrow R$ is $\alpha$ differentiable at $t_{0}>0,0<\alpha \leq 1$, then $f$ is continuous at $t_{0}$.

Theorem 2. [3]. Let $0<\alpha \leq 1$, and let $f, g$ be $\alpha$ differentiable at a point $t>0$. Then

(i) $T_{\alpha}(a f+b g)=a\left(T_{\alpha} f\right)+b\left(T_{\alpha} g\right), \forall a, b \in R$.

(ii) $T_{\alpha}\left(t^{p}\right)=p t^{p-\alpha}, \forall p \in R$.

(iii) $T_{\alpha}(\lambda)=0$, for all constant functions $f(t)=\lambda$.

(iv) $T_{\alpha}(f g)=f\left(T_{\alpha} g\right)+g\left(T_{\alpha} f\right)$

(v) $T_{\alpha}(f / g)=\left(g\left[T_{\alpha} f\right]+f\left[T_{\alpha} g\right]\right) / g^{2}$

(vi) If, in addition, $f$ is differentiable, then $\left(T_{\alpha} f\right)(t)=$ $t^{1-\alpha}(d f / d t)(t)$.

The conformable fractional derivative of certain functions for the above definition is given as:

(i) $T_{\alpha}(1)=0$

(ii) $T_{\alpha}(\sin (a t))=a t^{1-\alpha} \cos (a t)$,

(iii) $T_{\alpha}(\cos (a t))=-a t^{1-\alpha} \sin (a t)$,

(iv) $T_{\alpha}\left(e^{a t}\right)=a e^{a t}, a \in R$.

Definition 2. The (left) conformable derivative starting from a of a given function $f:[a, \infty) \rightarrow R$ of order $0<\alpha \leq 1$, [4], is defined by

$$
\left(T_{\alpha}^{a} f\right)(t)=\lim _{\varepsilon \rightarrow 0} \frac{f\left(t+\varepsilon(t-a)^{1-\alpha}\right)-f(t)}{\varepsilon},
$$

When $a=0$, it is written as $\left(T_{\alpha} f\right)(t)$. If $f$ is $\alpha$ differentiable in some $(a, b)$, then the following can be defined as:

$$
\left(T_{\alpha}^{a} f\right)(a)=\lim _{t \rightarrow a^{+}}\left(T_{\alpha}^{a} f\right)(a),
$$

Theorem 3 (Chain Rule). [4]. Assume $f, g:(a, \infty) \rightarrow R$ be (left) $\alpha$-differentiable functions, where $0<\alpha \leq 1$. By letting $h(t)=f(g(t)), h(t)$ is $\alpha$-differentiable for all $t \neq a$ and $g(t) \neq 0$, therefore, we have the following:

$$
\left(T_{\alpha}^{a} h\right)(t)=\left(T_{\alpha}^{a} f\right)(g(t)) \cdot\left(T_{\alpha}^{a} g\right)(t) \cdot(g(t))^{\alpha-1},
$$

$$
\text { if } t=a \text {, then }
$$

$$
\left(T_{\alpha}^{a} h\right)(a)=\lim _{t \rightarrow a^{+}}\left(T_{\alpha}^{a} f\right)(g(t)) \cdot\left(T_{\alpha}^{a} g\right)(t) \cdot(g(t))^{\alpha-1},
$$

Theorem 4 (Rolle's Theorem). [3]. Let $a>0, \alpha \in(0,1]$ and $f:[a, \infty) \rightarrow$ be a given function that satisfies the following:

- $f$ is continuous on $[a, b]$.

- $f$ is $\alpha$-differentiable on $(a, b)$.

- $f(a)=f(b)$.

Then, there exists $c \in(a, b)$, such that $\left(T_{\alpha} f\right)(c)=0$. Corollary 1. Let $I \subset[0, \infty), \alpha \in(0,1]$ and $f: I \rightarrow R$ be a given function that satisfies

- $f$ is a differentiable on $\mathbf{I}$.

- $f(a)=f(b)=0$ for certain $c \in I$

Then, there exists $c \in(a, b)$, such that $\left(T_{\alpha} f\right)(c)=0$. Theorem 5. (Mean Value Theorem). [3]. Let $a>0$, $\alpha \in(0,1]$ and $f:[a, \infty) \rightarrow R$ be a given function that satisfies

- $f$ is continuous in $[a, b]$.

- $f$ is $\alpha$-differentiable on $(a, b)$.

Then, exists $c \in(a, b)$ such that

$$
\left(T_{\alpha} f\right)(c)=\frac{f(b)-f(a)}{\frac{b^{\alpha}}{\alpha}-\frac{a^{\alpha}}{\alpha}},
$$

Theorem 6. [5]. Let $a>0, \alpha \in(0,1]$ and $f:[a, \infty) \rightarrow$ $R$ be a given function that satisfies

- $f$ is continuous in $[a, b]$.

- $f$ is $\alpha$-differentiable on $(a, b)$.

If $\left(T_{\alpha} f\right)(c)=0$ for all $t \in(a, b)$, then $f$ is a constant on $[a, b]$.

Corollary 7. [5]. Let $a>0, \alpha \in(0,1]$ and $F, G$ : $[a, \infty) \rightarrow R$ be functions such that $\left(T_{\alpha} F\right)(t)=\left(T_{\alpha} G\right)(t)$ for all $t \in(a, b)$. Then, there exists a constant $C$ such that

$$
F(t)=G(t)+C,
$$

The following definition is the $\alpha$-fractional integral of a function $f$ starting from $a \geq 0$ :

\section{Definition 3}

$$
I_{\alpha}^{a}(f)(t)=\int_{a}^{t} \frac{f(x)}{x^{1-\alpha}} \cdot d x,
$$


where the integral is the usual Riemann improper integral, and $\alpha \in(01]$ [2].

Theorem 8. $T_{\alpha}^{a} I_{\alpha}^{a}(f)(t)=f(t)$, for $t \geq a$, where $f$ is any continuous function in the domain of $I_{\alpha}$.

Lemma 9. Let $f:(a, b) \rightarrow R$ be differentiable and $\alpha \in(0,1]$. Then, for all $a>0$, we have [3],

$$
I_{\alpha}^{a} T_{\alpha}^{a}(f)(t)=f(t)-f(a),
$$

Finally, we give the definition of non-conformable $\alpha$ Wronskian, which is necessary in the next section.

Definition 4. Let $x$ and $y$ be given conformable $\alpha$ differentiable functions on $[a, b]$ with $a \geq 0$ and $\alpha \in(0,1]$. We set the following:

$$
W^{\alpha}(x, y)(t)=\left|\begin{array}{cc}
x(t) & y(t) \\
\left(T_{\alpha} x\right)(t) & \left(T_{\alpha} y\right)(t)
\end{array}\right| .
$$

\section{Sturm's theorems}

In this section, we consider the scalar fractional differential equation of order $\alpha+\alpha$ as follows:

$$
T_{\alpha} T_{\alpha} x(t)+p(t) T_{\alpha} x(t)+q(t) x(t)=0,
$$

with continuous functions $p$ and $q$, and $\alpha \in(0,1]$. Traditionally, from [19], two functions $x$ and $y$ that are continuous on $[a, b]$ for some $0 \leq a<b$, will be called linearly dependent if there exist $c_{1}, c_{2} \in R$ such that $\left|c_{1}\right|+\left|c_{1}\right|>0$ and $c_{1} x(t)+c_{2} y(t) \equiv 0$ for all $t \in[a, b]$. In the other case, they are linearly independent.

Remark 1. We can write

$$
W^{\alpha}(x, y)(t)=e^{-\int_{t_{0}}^{t} \frac{p(x)}{x^{1-\alpha}} d x} W^{\alpha}(x, y)\left(t_{0}\right),
$$

for two solutions $x$ and $y$ of [5] and some $t_{0} \in(a, b)$. In fact, we apply the operator $T_{\alpha}$ on $W^{\alpha}(x, y)(t)$ to obtain

$$
\begin{aligned}
T_{\alpha}\left(W^{\alpha}(x, y)(t)\right) & =T_{\alpha}\left(x(t) T_{\alpha} y(t)-y(t) T_{\alpha} x(t)\right. \\
& =T_{\alpha} x(t) T_{\alpha} y(t)+x(t) T_{\alpha} T_{\alpha} y(t) \\
& -T_{\alpha} y(t) T_{\alpha} x(t)-y(t) T_{\alpha} T_{\alpha} x(t) .
\end{aligned}
$$

However, $x$ and $y$ satisfies (11). Hence, we have:

$$
T_{\alpha} T_{\alpha} x(t)=-p(t) T_{\alpha} x(t)-q(t) x(t),
$$

and

$$
T_{\alpha} T_{\alpha} y(t)=-p(t) T_{\alpha} y(t)-q(t) y(t)
$$

Therefore, we get

$$
\begin{aligned}
T_{\alpha}\left(W^{\alpha}(x, y)(t)\right) & =-\left(x(t) T_{\alpha} y(t)-y(t) T_{\alpha} x(t)\right) p(t) \\
& =-\left(W^{\alpha}(x, y)(t)\right) p(t) .
\end{aligned}
$$

Thus

$$
\frac{T_{\alpha}\left(W^{\alpha}(x, y)(t)\right)}{W^{\alpha}(x, y)(t)}=-p(t) .
$$

Consequently, we have

$$
W^{\alpha}(x, y)(t)=e^{-\int_{t_{0}}^{t}(p(x) / 1-\alpha) d x} W^{\alpha}(x, y)\left(t_{0}\right) .
$$

This completes the proof.

Similar to the classical case, by using the above formula, we can immediately obtain the following equivalent condition of linear independence:

Theorem 10. Two solutions $x$ and y of Eq. (11) defined on $[a, b]$ for some $0 \leq a<b$ are linearly independent if and only if $W^{\alpha}(x, y)(t) \neq 0$ for all $t \in[a, b]$.

Now, we propose a conformable version of three classical results, the Sturm-Picone identity, Sturm's comparison, and separation theorems of order $\alpha+\alpha$ [20].

Let us now introduce the conformable self-adjoint SturmLiouville equation as follows:

$$
\begin{aligned}
& -T_{\alpha}\left(p_{1}(t) T_{\alpha} x(t)\right)+p_{0}(t) x(t)=0, \\
& -T_{\alpha}\left(p_{1}(t) T_{\alpha} x(t)\right)+p_{0}(t) x(t)=0,
\end{aligned}
$$

where $p_{0}, p_{1}, q_{0}, q_{1}, T_{\alpha} p_{1}, T_{\alpha} q_{1}$ are continuous on some closed interval $I \subset[0,+\infty), p_{1}>0, q_{1}>0$ on $I$ and $\alpha \in(0,1]$.

Theorem 11 (Conformable Picone Identity). If $x(t)$, $y(t)$ and $p_{1}(t) T_{\alpha} x(t), q_{1}(t) T_{\alpha} y(t)$ are $\alpha$-differentiable for $t \in I$ and $y(t) \neq 0$ in $I$, then we obtain

$$
\begin{aligned}
& T_{\alpha}\left(\frac{x(t)}{y(x)}\left[p_{1}(t) y(t) T_{\alpha} x(t)-q_{1}(t) x(t) T_{\alpha} y(t)\right]\right) \\
& \quad=x(t) T_{\alpha}\left(p_{1}(t) T_{\alpha} x(t)\right)-\frac{(x(t))^{2}}{y(t)} T_{\alpha}\left(q_{1}(t) T_{\alpha} y(t)\right) \\
& \quad+\left(p_{1}(t)-q_{1}(t)\right)\left(T_{\alpha} x(t)\right)^{2}+q_{1}(t) \\
& \quad \times\left(T_{\alpha} x(t)-\frac{x(t)}{y(t)} T_{\alpha} y(t)\right)^{2} .
\end{aligned}
$$

Proof. This arises from the straightforward $\alpha$ differentiation.

Theorem 12 (Conformable Sturm's Comparison Theorem). Let 0 ? $a<b$ be two consecutive zeros of a nontrivial solution $x(t)$ of Eq. (3.3). Suppose that

(i) $0<q_{1}(t) \leq p_{1}(t)$,

and

(ii) $q_{0}(t) \leq p_{0}(t)$,

for all $t \in[a, b]$. Then, every solution $y(t)$ of Eq. (14) has at least one zero in the closed interval $[a, b]$.

Proof. If $x(t)$ and $y(t)$ are solutions of (13) and (14), respectively, and $y(t) \neq 0$ for all $t \in[a, b]$, then the 
conformable Picone identity (15) yields on substitution of Eq. (13) and (14) as follows:

$$
\begin{aligned}
T_{\alpha} & \left(\frac{x(t)}{y(t)}\left[p_{1}(t) y(t) T_{\alpha} x(t)-q_{1}(t) x(t) T_{\alpha} y(t)\right]\right) \\
& =\left(p_{0}(t)-q_{0}(t)\right)(x(t))^{2}+\left(p_{1}(t)-q_{1}(t)\right)\left(T_{\alpha} x(t)\right)^{2} \\
& +q_{1}(t)\left(T_{\alpha} x(t)-\frac{x(t)}{y(t)} T_{\alpha} y(t)\right)^{2} .
\end{aligned}
$$

Integrating over $[a, b]$; therefore, we have (see Lemma 9),

$$
\begin{aligned}
& \int_{a}^{b}\left(\left(p_{0}(t)-q_{0}(t)\right)(x(t))^{2}+\left(p_{1}(t)-q_{1}(t)\right)\left(T_{\alpha} x(t)\right)^{2}\right. \\
& \left.\quad+q_{1}(t)\left[T_{\alpha} x(t)-\frac{x(t)}{y(t)} T_{\alpha} y(t)\right]^{2}\right) \frac{1}{t^{1-\alpha}} d t \\
& \quad=\left[\frac{x(t)}{y(t)}\left[p_{1}(t) y(t) T_{\alpha} x(t)-q_{1}(t) x(t) T_{\alpha} y(t)\right]_{t=a}^{t=b} .\right.
\end{aligned}
$$

The right-hand side of Eq. (16) evaluates to zero by assuming $x(a)=x(b)=0$, and $y(a) \neq 0, y(b) \neq 0$. Since $q_{1}(t)>0$ in $[a, b]$, the third term of the integrand is nonnegative over $[a, b]$. Hence, we must have either

(i) $T_{\alpha} x(t)-(x(t) / y(t)) T_{\alpha} y(t) \equiv 0$ in $[a, b]$

or

(ii)

$$
\begin{aligned}
& \int_{a}^{b}\left(\left[p_{0}(t)-q_{0}(t)\right](x(t))^{2}+\left[p_{1}(t)-q_{1}(t)\right]\left(T_{\alpha} x(t)\right)^{2}\right) \\
& \quad \times\left(1 / t^{1-\alpha}\right) d t<0 .
\end{aligned}
$$

However, case (ii) gives an immediate contradiction since $p_{0}(t)-q_{0}(t) \geq 0$ and $p_{1}(t)-q_{1}(t) \geq 0$ by assumption. In Case (i), we are also led to a contradiction since (i) implies

$$
\frac{y(t) T_{\alpha} x(t)-x(t) T_{\alpha} y(t)}{(y(t))^{2}}=T_{\alpha}\left(\frac{x(t)}{y(t)}\right) \equiv 0,
$$

or $(t) \equiv k y(t)$ for all $t \in[a, b]$, for some $k \neq 0$, but $y(a)=y(b)=0$ which is a contrary to our assumption.

Theorem 13 (Conformable Sturm's Separation Theorem). Let $0 \leq a<b$ be two consecutive zeros of a nontrivial solution $x(t)$ of Eq. (13). Let $y(t)$ be any other solution of $E q$. (13) which is linearly independent of $x(t)$. Then, $y(t)$ has exactly one zero of the interval $(a, b)$. In other words, the zeros of any two linearly independent solutions of (13) are interlaced.

Proof. On the contrary, suppose that $y(t) \neq 0$ for all $t \in(a, b)$. Since $x(t)$ and $y(t)$ are linearly independent, it follows that $y(a) \neq 0$; otherwise, we would have

$$
W^{\alpha}(x, y)(a)=\left|\begin{array}{cc}
x(a) & y(a) \\
T_{\alpha} x(t) & T_{\alpha} y(t)
\end{array}\right|=0,
$$

which implies that the conformable Wronskian, $W^{\alpha}(x, y)(t)$, is zero for all $t$ and that $x(t)$ and $y(t)$ are linearly dependent. For the same reason, we know that $y(b) \neq 0$, but when $q_{1}(t) \equiv p_{1}(t)$ and $q_{0}(t) \equiv p_{0}(t)$, Eq. (16) becomes

$$
\begin{aligned}
& \int_{a}^{b} p_{1}(t)\left(T_{\alpha} x(t)-\frac{x(t)}{y(t)} T_{\alpha} y(t)\right)^{2} \frac{1}{t^{1-\alpha}} d t \\
& =\left[\frac{x(t)}{y(t)} p_{1}(t)\left(y(t) T_{\alpha} x(t)-x(t) T_{\alpha} y(t)\right)\right]_{t=a}^{t=b} .
\end{aligned}
$$

Since $y(a) \neq 0$ and $y(b) \neq 0$, the right-hand side evaluates to zero. Since $p_{1}(t)>0$ in $[a, b]$, it follows that $T_{\alpha} x(t)-(x(t) / y(t)) T_{\alpha} y(t) \equiv 0$, or

$$
W^{\alpha}(x, y)(t)=y(t) T_{\alpha} x(t)-x(t) T_{\alpha} y(t) \equiv 0,
$$

for all $t \in(a, b)$. Hence, $x(t)$ and $y(t)$ are linearly dependent on $(a, b)$ which is a contrary to our assumption.

\section{Remark 2.}

(i) Conformable Sturm's Comparison Theorem guarantees the existence of at least one zero.

(ii) The assumption $q_{0}(t) \leq p_{0}(t)$ cannot be dropped. Consider the equation on $t \geq 0, T_{\alpha} T_{\alpha} x(t)+x(t)=$ $0\left(p_{1}(t)=1, p_{0}(t)=-1\right)$ and $T_{\alpha} T_{\alpha} y(t)-y(t)=$ $0\left(q_{1}(t)=1, q_{0}(t)=1\right)$ and let $x(t)$ and $y(t)$ be their non-trivial solutions, respectively. Between any two zeros of $x(t), y(t)$ does not admit a zero.

(iii) Consider the equation on $t \geq 0, T_{\alpha} T_{\alpha} x(t)+x(t)=$ $0\left(p_{1}(t)=1, p_{0}(t)=-1\right)$ and $T_{\alpha} T_{\alpha} y(t)+4 y(t)=$ $0\left(q_{1}(t)=1, q_{0}(t)=-4\right)$, and let $x(t)=\sin \left(t^{\alpha} / \alpha\right)$ and $y(t)=\sin \left(2 t^{\alpha} / \alpha\right)$ be their non-trivial solutions, respectively. However, there is no zero of $x(t)$ between two consecutive zeros of $y(t)$.

Remark 3. An important application of Sturm's Comparison Theorem is to provide a good understanding of the zero set on non-trivial solutions of Conformable Bessel's Equation. The Conformable Bessel's Equation is given by

$$
t^{2 \alpha} T_{\alpha} T_{\alpha} y(t)+\alpha t^{\alpha} T_{\alpha} y(t)+\alpha^{2}\left(t^{2 \alpha}-p^{2}\right) y(t)=0,
$$

where $\alpha \in(0,1]$ and $p \geq 0$. Clearly, if $\alpha=0$, the above equation is just the classical Bessel Equation, [19]. For more information about the conformable Bessel's function in the solution of wave equation, we refer to [21].

For $t>0$, making a change variable $y=v / t^{\alpha / 2}$, the Eq. (17) transforms into

$$
T_{\alpha} T_{\alpha} y(t)+\alpha^{2}\left(1+\frac{1-4 p^{2}}{4 t^{2 \alpha}}\right) v(t)=0 .
$$


(To obtain the above equation, we start differentiating the equation $t^{\alpha / 2} y=v$ ).

Case 1: $p>1 / 2$. In this case, compare (18) with

$$
T_{\alpha} T_{\alpha} y(t)+\alpha^{2} y(t)=0,
$$

which has a solution $\sin \left(t^{\alpha}\right)$ with zeros at $t=(n \pi)^{1 / \alpha}$, $n \in N$. Therefore, a solution of (18) has at least one zero on each of the open interval $\left([\{n-1\} \pi]^{1 / \alpha},(n \pi)^{1 / \alpha}\right], n \in N$. Case 1: : $0<p<1 / 2$. In this case, compare (18) with

$$
T_{\alpha} T_{\alpha} y(t)+\alpha^{2} y(t)=0
$$

and conclude that between any two consecutives zeros, $a$ and $b$ of $v(t)$, there exists one zero of $\sin \left(t^{\alpha}\right)$. Thus, we have $a<(n \pi)^{1 / \alpha}<b$ for some $n \in N$.

\section{The study of conformable Green's Func- tions}

\subsection{Conformable Green's Functions}

In this section, we consider the conformable Sturm- Liouville system

$$
\left.\begin{array}{cc}
T_{\alpha}\left(p(t) T_{\alpha} x(t)\right)+(\lambda \rho(t)-q(t)) x(t)=0 & (19 a) \\
a_{1} x(a)+a_{2} T_{\alpha} x(a)=0 & (19 b) \\
b_{1} x(a)+b_{2} T_{\alpha} x(a)=0 & (19 c)
\end{array}\right\},
$$

with continuous functions $p(t), q(t)$ and $\rho(t)$ on $[a, b]$ for some $0 \leq a<b$, such that $\rho(t) \geq 0$ and $p(t) \geq 0$ for all $t \in[a, b]$ and $\alpha \in(0,1]$.

Definition 5. Let $Q$ denote the square $Q=[a, b] \times[a, b]$ for some $0 \leq a<b$, in the $t \in$-plane. A function $G^{\alpha}(t, \varepsilon)$ defined in $Q$ is called conformable Green's Function of SturmLiouville system (19), if it has the following properties:

(i) The function $G^{\alpha}(t, \varepsilon)$ is continuous in $Q$.

(ii) Let $\varepsilon \in(a, b)$ be fixed. Then, $G^{\alpha}(t, \varepsilon)$ has conformable partial derivatives of left and right with respect to variable $t$, for $t=\varepsilon$, and it is verified as follows:

$$
\frac{\partial^{\alpha}}{\partial t^{\alpha}} G^{\alpha}\left(\varepsilon^{+}, \varepsilon\right)-\frac{\partial^{\alpha}}{\partial t^{\alpha}} G^{\alpha}\left(\varepsilon^{-}, \varepsilon\right)=-\frac{1}{p(\varepsilon)} .
$$

(iii) Let $\varepsilon \in[a, b]$ be fixed. Then, $G^{\alpha}(t, \varepsilon)$ has continuous conformable partial derivatives of first and second order with respect to variable $t$, if $t \neq \varepsilon$, and it is verified as follows:

$$
\frac{\partial^{\alpha}}{\partial t^{\alpha}}\left(p(t) T_{\alpha} G^{\alpha}(t, \varepsilon)\right)+(\lambda \rho(t)-q(t)) G^{\alpha}(t, \varepsilon)=0 .
$$

(iv) Let $\varepsilon \in(a, b)$ be fixed. Then, $G^{\alpha}(t, \varepsilon)$ satisfies the boundary conditions (19b) and (19c).
Theorem 14. Let $x_{1}(t)$ and $x_{2}(t)$ be two solutions of (19a) that verify condition $(19 b)$. Then, $x_{1}(t)$ and $x_{2}(t)$ are linearly dependent.

Proof. Since $\left|a_{1}\right|+\left|a_{2}\right| \neq 0$, it follows from

$$
\begin{aligned}
& a_{1} x_{1}(a)+a_{2} T_{\alpha} x_{1}(a)=0, \\
& a_{1} x_{1}(a)+a_{2} T_{\alpha} x_{1}(a)=0,
\end{aligned}
$$

that

$$
W^{\alpha}(x, y)(a)=\left|\begin{array}{cc}
x_{1}(a) & x_{2}(a) \\
T_{\alpha} x_{1}(a) & T_{\alpha} x_{2}(a)
\end{array}\right|=0
$$

Therefore, $x_{1}(t)$ and $x_{2}(t)$ are linearly dependent.

Theorem 15. Let $x_{1}(t)$ and $x_{2}(t)$ be two solutions of (19a) that verify condition $(19 c)$. Then, $x_{1}(t)$ and $x_{2}(t)$ are linearly dependent.

Proof. It is analogous to the proof of the above theorem.

Theorem 16. System (19) has no Green's Function if $\lambda$ is an eigenvalue.

Proof. Let $x_{1}(t)$ be an eigenfunction of system (19). Let $x_{2}(t)$ be a solution of (19a) linearly independent of $x_{1}(t)$. From Theorems 14 and 15, it turns out that $x_{2}(t)$ does not verify the conditions (19b) and (19c).

According to the condition (iii) of $G^{\alpha}(t, \varepsilon)$, the said function is a solution of (19a) in the intervals $a \leq t<\varepsilon$ and $\varepsilon<t \leq b$, so it has the following form:

$$
G^{\alpha}(t, \varepsilon)=\left\{\begin{array}{ll}
A_{1}(\varepsilon) x_{1}(t)+A_{2}(\varepsilon) x_{2}(t) & a \leq t<\varepsilon \\
B_{1}(\varepsilon) x_{1}(t)+B_{2}(\varepsilon) x_{2}(t) & \varepsilon<t \leq b
\end{array} .\right.
$$

Let us now express that $G^{\alpha}(t, \varepsilon)$ meets the condition (iv)

$$
\begin{aligned}
& a_{1}\left(A_{1}(\varepsilon) x_{1}(a)+A_{2}(\varepsilon) x_{2}(a)\right) \\
& \quad+a_{2}\left(A_{1}(\varepsilon) T_{\alpha} x_{1}(a)+A_{2}(\varepsilon) T_{\alpha} x_{2}(a)\right)=0, \\
& b_{1}\left(B_{1}(\varepsilon) x_{1}(b)+B_{2}(\varepsilon) x_{2}(b)\right) \\
& \quad+b_{2}\left(B_{1}(\varepsilon) T_{\alpha} x_{1}(b)+B_{2}(\varepsilon) T_{\alpha} x_{2}(b)\right)=0 .
\end{aligned}
$$

Since $x_{1}(t)$ meets both conditions (19b) and (19c), the above equalities are reduced to

$$
\begin{gathered}
A_{2}(\varepsilon)\left(a_{1} x_{2}(a)+a_{2} T_{\alpha} x_{2}(a)\right)=0, \\
B_{2}(\varepsilon)\left(b_{1} x_{2}(b)+b_{2} T_{\alpha} x_{2}(b)\right)=0 .
\end{gathered}
$$

On the contrary, we have

$$
\begin{gathered}
a_{1} x_{2}(a)+a_{2} T_{\alpha} x_{2}(a) \neq 0, \\
b_{1} x_{2}(b)+b_{2} T_{\alpha} x_{2}(b) \neq 0 .
\end{gathered}
$$

so that

$$
\begin{aligned}
& A_{2}(\varepsilon)=0, \quad a \leq t<\varepsilon, \\
& B_{2}(\varepsilon)=0, \quad \varepsilon<t \leq b .
\end{aligned}
$$


From here, we have

$$
G^{\alpha}(t, \varepsilon)=\left\{\begin{array}{ll}
A_{1}(\varepsilon) x_{1}(t) & a \leq t<\varepsilon \\
B_{1}(\varepsilon) x_{1}(t) & \varepsilon<t \leq b
\end{array} .\right.
$$

Since $G^{\alpha}(t, \varepsilon)$ is a continuous function, we obtain

$$
\begin{aligned}
& \lim _{t \rightarrow \varepsilon^{-}} G^{\alpha}(t, \varepsilon)=A_{1}(\varepsilon) x_{1}(\varepsilon) \\
& =\lim _{t \rightarrow \varepsilon^{+}} G^{\alpha}(t, \varepsilon)=B_{1}(\varepsilon) x_{1}(\varepsilon),
\end{aligned}
$$

so that

$$
A_{1}(\varepsilon)=B_{1}(\varepsilon), \quad a<\varepsilon<b .
$$

From here, it follows that

$$
\frac{\partial^{\alpha}}{\partial t^{\alpha}} G^{\alpha}\left(\varepsilon^{+}, \varepsilon\right)-\frac{\partial^{\alpha}}{\partial t^{\alpha}} G^{\alpha}\left(\varepsilon^{-}, \varepsilon\right)=0
$$

which contradicts condition (ii).

Theorem 17. System (19) has one, and only one, Green's Function if $\lambda$ is not an eigenvalue.

Proof. Let $x_{1}(t)$ and $x_{2}(t)$ two solutions of (19) such that

$$
\begin{gathered}
x_{1}(a)=a_{2}, \quad T_{\alpha} x_{1}(a)=-a_{1}, \\
x_{2}(b)=b_{2}, \quad T_{\alpha} x_{2}(b)=-b_{1} .
\end{gathered}
$$

Since $\left|a_{1}\right|+\left|a_{2}\right| \neq 0,\left|b_{1}\right|+\left|b_{2}\right| \neq 0, x_{1}(t)$ and $x_{2}(t)$ are not null, they are also satisfying conditions (19b) and (19c), respectively.

These solutions are linearly independent, since otherwise it would be

$$
x_{1}(t)=\mu x_{2}(t), \quad \mu \neq 0 .
$$

Therefore, we have

$$
b_{1} x_{1}(b)+b_{2} T_{\alpha} x_{1}(b)=\mu\left[b_{1} x_{2}(b)+b_{2} T_{\alpha} x_{2}(b)\right]=0 .
$$

As a result, $x_{1}(t)$ would comply with (19b) and (19c). This is not possible since $x_{1}(t)$ is not an eigenfunction.

The reasoning as in the proof of Theorem 16, we have to

$$
G^{\alpha}(t, \varepsilon)=\left\{\begin{array}{ll}
A_{1}(\varepsilon) x_{1}(t)+A_{2}(\varepsilon) x_{2}(t) & a \leq t<\varepsilon \\
B_{1}(\varepsilon) x_{1}(t)+B_{2}(\varepsilon) x_{2}(t) & \varepsilon<t \leq b
\end{array} .\right.
$$

Expressing that $G^{\alpha}(t, \varepsilon)$ meets the condition (iv), and it turns out that

$$
\begin{aligned}
& a_{1}\left(A_{1}(\varepsilon) x_{1}(a)+A_{2}(\varepsilon) x_{2}(a)\right) \\
& \quad+a_{2}\left(A_{1}(\varepsilon) T_{\alpha} x_{1}(a)+A_{2}(\varepsilon) T_{\alpha} x_{2}(a)\right)=0, \\
& b_{1}\left(B_{1}(\varepsilon) x_{1}(b)+B_{2}(\varepsilon) x_{2}(b)\right) \\
& \quad+b_{2}\left(B_{1}(\varepsilon) T_{\alpha} x_{1}(b)+B_{2}(\varepsilon) T_{\alpha} x_{2}(b)\right)=0,
\end{aligned}
$$

that is reduced to

$$
\begin{gathered}
A_{2}(\varepsilon)\left(a_{1} x_{2}(a)+a_{2} T_{\alpha} x_{2}(a)\right)=0, \\
B_{1}(\varepsilon)\left(b_{1} x_{1}(b)+b_{2} T_{\alpha} x_{1}(b)\right)=0,
\end{gathered}
$$

from where it follows, remembering that $x_{1}(t)$ and $x_{2}(t)$ are not eigenfunctions and, therefore, $a_{1} x_{2}(a)+a_{2} T_{\alpha} x_{2}(a) \neq 0$, $b_{1} x_{1}(b)+b_{2} T_{\alpha} x_{1}(b) \neq 0$,

$$
\left.\begin{array}{l}
A_{2}(\varepsilon)=0 \\
B_{1}(\varepsilon)=0
\end{array}\right\} \quad a<\varepsilon<b .
$$

Now, by applying conditions (i) and (ii), it turns out that

$$
A_{1}(\varepsilon) x_{1}(\varepsilon)+B_{2}(\varepsilon) x_{2}(\varepsilon)=0,
$$

$$
A_{1}(\varepsilon) T_{\alpha} x_{1}(\varepsilon)+B_{2}(\varepsilon) T_{\alpha} x_{2}(\varepsilon)=\frac{1}{p(\varepsilon)},
$$

which allows us to calculate the following:

$$
\begin{aligned}
& A_{1}(\varepsilon)=\frac{-x_{2}(\varepsilon)}{p(\varepsilon)\left[x_{1}(\varepsilon) T_{\alpha} x_{2}(\varepsilon)-x_{2}(\varepsilon) T_{\alpha} x_{1}(\varepsilon)\right]} \\
& B_{2}(\varepsilon)=\frac{-x_{1}(\varepsilon)}{p(\varepsilon)\left[x_{1}(\varepsilon) T_{\alpha} x_{2}(\varepsilon)-x_{2}(\varepsilon) T_{\alpha} x_{1}(\varepsilon)\right]}
\end{aligned}
$$

Note that $x_{1}(\varepsilon) T_{\alpha} x_{2}(\varepsilon)-x_{2}(\varepsilon) T_{\alpha} x_{1}(\varepsilon)$ is nonzero since it is conformable Wronskian of two linearly independent solutions of Eq. (19).

Given the following:

$$
\begin{aligned}
& T_{\alpha}\left(p(t) T_{\alpha} x_{1}(t)\right)+(\lambda \rho(t)-q(t)) x_{1}(t)=0 \\
& T_{\alpha}\left(p(t) T_{\alpha} x_{2}(t)\right)+(\lambda \rho(t)-q(t)) x_{2}(t)=0 .
\end{aligned}
$$

By multiplying the first equation by $x_{2}(t)$, the second by $x_{1}(t)$, and subtracting, we have

$$
x_{2}(t) T_{\alpha}\left(p(t) T_{\alpha} x_{1}(t)\right)-x_{1}(t) T_{\alpha}\left(p(t) T_{\alpha} x_{2}(t)\right)=0
$$

that can be written in the form

$$
\left.p(t)\left(x_{2}(t) T_{\alpha} x_{1}(t)\right)-x_{1}(t) T_{\alpha} x_{2}(t)\right)=0 .
$$

So, $p(\varepsilon)\left(x_{2}(\varepsilon) T_{\alpha} x_{1}(\varepsilon)-x_{1}(\varepsilon) T_{\alpha} x_{2}(\varepsilon)\right)$ is a constant $K$ that does not depend on $\varepsilon$.

Hence, we have

$$
G^{\alpha}(t, \varepsilon)=\left\{\begin{array}{cc}
\frac{1}{K} x_{1}(t) x_{2}(\varepsilon) & a \leq t<\varepsilon \\
\frac{1}{K} x_{1}(\varepsilon) x_{2}(t) & \varepsilon<t \leq b
\end{array}\right.
$$

The conformable Green's Function $G^{\alpha}(t, \varepsilon)$ has the properties (i) - (iv). The uniqueness of this function is easily deduced from the method that we have followed to determine $G^{\alpha}(t, \varepsilon)$.

Ejemple 1. Consider the system

$$
\left.\begin{array}{rl}
T_{\alpha} T_{\alpha} x(t)+x(t) & =0 \quad t \in\left[0,(\alpha \pi)^{1 / \alpha}\right] \\
x(0)+T_{\alpha} x(0) & =0 \\
x\left((\alpha \pi)^{1 / \alpha}\right) & =0
\end{array}\right\}
$$

for some $\alpha \in(0,1]$, we will find the corresponding conformable Green's Function. In this case $p(t)=1, q(t)=$ 
$-1, \lambda=0, \rho(t)$ is any positive continuous function in $\left.\left[0,(\alpha \pi)^{(} 1 / \alpha\right)\right], a_{1}=1, a_{2}=1, b_{1}=1, b_{2}=0$.

The general solution of $T_{\alpha} T_{\alpha} x(t)+x(t)=0$ is

$$
x(t)=A \cos \frac{t^{\alpha}}{\alpha}+B \sin \frac{t^{\alpha}}{\alpha} .
$$

Then, we have

$$
\begin{gathered}
x(0)+T_{\alpha} x(0)=A+B=0, \\
x\left((\alpha \pi)^{1 / \alpha}\right)=-A=0 .
\end{gathered}
$$

From here $A=0, B=0$, so there was the conformable Green's Function of the given system.

The solutions of $T_{\alpha} T_{\alpha} x(t)+x(t)=0 ; x_{1}(t)=$ $\cos t^{\alpha} / \alpha+\sin t^{\alpha} / \alpha, x_{2}(t)=\sin t^{\alpha} / \alpha$ satisfy the conditions $\left.x(0)+T_{\alpha} x(0)=0, x\left((\alpha \pi)^{(} 1 / \alpha\right)\right)=0$. The conformable Green's Function has the form

$$
G^{\alpha}(t, \varepsilon)=\left\{\begin{array}{cc}
\frac{1}{K} x_{2}(\varepsilon) x_{1}(t) & 0 \leq t<\varepsilon \\
\frac{1}{K} x_{1}(\varepsilon) x_{2}(t) & \varepsilon<t \leq(\alpha \pi)^{1 / \alpha}
\end{array}\right.
$$

so that

$$
\begin{aligned}
K & =p(\varepsilon)\left(x_{2}(\varepsilon) T_{\alpha} x_{1}(\varepsilon)-x_{1}(\varepsilon) T_{\alpha} x_{2}(\varepsilon)\right) \\
& =\left(-\sin \frac{\varepsilon^{\alpha}}{\alpha}+\cos \frac{\varepsilon^{\alpha}}{\alpha}\right) \sin \frac{\varepsilon^{\alpha}}{\alpha} \\
& -\left(-\cos \frac{\varepsilon^{\alpha}}{\alpha}+\sin \frac{\varepsilon^{\alpha}}{\alpha}\right) \cos \frac{\varepsilon^{\alpha}}{\alpha}=-1 .
\end{aligned}
$$

Therefore, we obtain

$$
\begin{aligned}
& G^{\alpha}(t, \varepsilon) \\
& =\left\{\begin{array}{lc}
-\sin \frac{\varepsilon^{\alpha}}{\alpha}\left(\cos \frac{t^{\alpha}}{\alpha}+\sin \frac{t^{\alpha}}{\alpha}\right) & 0 \leq t<\varepsilon \\
-\left(\cos \frac{\varepsilon^{\alpha}}{\alpha}+\sin \frac{\varepsilon^{\alpha}}{\alpha}\right) \sin \frac{t^{\alpha}}{\alpha} & \varepsilon<t \leq(\alpha \pi)^{1 / \alpha}
\end{array}\right.
\end{aligned}
$$

\subsection{The applicability of conformable Green's function}

In this section, we consider the system

$$
\begin{gathered}
T_{\alpha}\left(p(t) T_{\alpha} x(t)\right)-q(t) x(t)=0 \\
a_{1} x(a)+a_{2}(x) T_{\alpha} x(a)=0 \\
b_{1} x(a)+b_{2}(x) T_{\alpha} x(b)=0
\end{gathered}
$$

obtained from (19) for $\lambda=0$. We now propose to solve the inhomogeneous system

$$
\begin{gathered}
T_{\alpha}\left(p(t) T_{\alpha} x(t)\right)-q(t) x(t)=-f(t) \\
a_{1} x(a)+a_{2}(x) T_{\alpha} x(a)=0 \\
b_{1} x(a)+b_{2}(x) T_{\alpha} x(b)=0
\end{gathered}
$$

where $f(t)$ is a real continuous function in the interval $[a, b]$ for some $0 \leq a<b$.

Theorem 18. If the homogeneous system (20) has its only solution as the identically null function, then (21) has only one solution, which is given by

$$
x(t)=\int_{a}^{b} G^{\alpha}(t, \varepsilon) f(\varepsilon) \frac{1}{\varepsilon^{1-\alpha}} d \varepsilon,
$$

where $G^{\alpha}(t, \varepsilon)$ is the conformable Green's Function of (20). Proof. That homogeneous system (20) has its unique solution as the identically null function which is equivalent to saying that $\lambda=0$ is not an eigenvalue of (19); therefore, there is the conformable Green's Function of (20).

Let $x_{1}(t)$ and $x_{2}(t)$ be two linearly independent solutions of (20a) that verify (20b) and (20c), respectively. Let us apply the conformable version of the method of variation of the parameters to solve (20a). Then, we have

$$
\begin{aligned}
x(t) & =A(t) x_{1}(t)+B(t) x_{2}(t) \\
T_{\alpha} & \left(p ( t ) \left[x_{1}(t) T_{\alpha} A(t)+x_{2}(t) T_{\alpha} B(t)+A(t) T_{\alpha} x_{1}(t)\right.\right. \\
& \left.\left.+B(t) T_{\alpha} x_{2}(t)\right]\right)-Q(t)\left(A(t) x_{1}(t)+B(t) x_{2}(t)\right) \\
& =-f(t),
\end{aligned}
$$

that is to say

$$
\begin{aligned}
A(t) & T_{\alpha}\left(p(t) T_{\alpha} x_{1}(t)-A(t) q(t) x_{1}(t)\right] \\
& +B(t) T_{\alpha}\left(p(t) T_{\alpha} x_{2}(t)-B(t) q(t) x_{2}(t)\right) \\
& +p(t)\left(T_{\alpha} A(t) T_{\alpha} x_{1}(t)+T_{\alpha} B(t) T_{\alpha} x_{2}(t)\right) \\
& +T_{\alpha}\left(p(t)\left[x_{1}(t) T_{\alpha} A(t)+x_{2}(t) T_{\alpha} B(t)\right]\right)=-f(t),
\end{aligned}
$$

that is

$$
\begin{aligned}
p(t) & \left(T_{\alpha} A(t) T_{\alpha} x_{1}(t)+T_{\alpha} B(t) T_{\alpha} x_{2}(t)\right) \\
& +T_{\alpha}\left(p(t)\left[x_{1}(t) T_{\alpha} A(t)+x_{2}(t) T_{\alpha} B(t)\right]\right)=-f(t) .
\end{aligned}
$$

We make

$$
x_{1}(t) T_{\alpha} A(t)+x_{2}(t) T_{\alpha} B(t)=0,
$$

and we have

$$
p(t)\left(T_{\alpha} A(t) T_{\alpha} x_{1}(t)+T_{\alpha} B(t) T_{\alpha} x_{2}(t)\right)=-f(t)
$$

so that

$$
\begin{aligned}
& T_{\alpha} A(t)=\frac{-x_{2}(t) f(t)}{p(t)\left(x_{2}(t) T_{\alpha} x_{1}(t)+x_{1}(t) T_{\alpha} x_{2}(t)\right)}, \\
& T_{\alpha} B(t)=\frac{-x_{1}(t) f(t)}{p(t)\left(x_{2}(t) T_{\alpha} x_{1}(t)+x_{1}(t) T_{\alpha} x_{2}(t)\right)} .
\end{aligned}
$$

We know, from the proof of Theorem 17, that $p(t)\left(x_{2}(t) T_{\alpha} x_{1}(t)+x_{1}(t) T_{\alpha} x_{2}(t)\right)$ is a constant, and it is equal to $K$. On the contrary, we have

$$
\begin{aligned}
& a_{1} x(a)+a_{2} T_{\alpha} x(a)=a_{1}\left(A(a) x_{1}(a)+B(a) x_{2}(a)\right) \\
& \quad+a_{2}\left(x_{1}(a) T_{\alpha} A(a)+x_{2}(a) T_{\alpha} B(a)+A(a) T_{\alpha} x_{1}(a)\right. \\
& \left.\quad+B(a) T_{\alpha} x_{2}(a)\right)=A(a)\left(a_{1} x_{1}(a)+a_{2} T_{\alpha} x_{1}(a)\right) \\
& \quad+B(a)\left(a_{1} x_{2}(a)+a_{2} T_{\alpha} x_{2}(a)\right)=B(a)\left(a_{1} x_{2}(a)\right. \\
& \left.\quad+a_{2} T_{\alpha} x_{2}(a)\right)=0
\end{aligned}
$$


and since $x_{2}(t)$ is not an eigenfunction of (20) it turns out that

$$
a_{1} x_{2}(a)+a_{2} T_{\alpha} x_{2}(a) \neq 0,
$$

so that $B(a)=0$.

By writing now the following:

$$
b_{1} x(b)+b T_{\alpha} x(a)=0 .
$$

Similarly, we obtain $A(b)=0$.

So, we have

$$
A(t)=\int_{a}^{t} \frac{x_{2}(\varepsilon)}{K} f(\varepsilon) \frac{1}{\varepsilon^{1-\alpha}} d \varepsilon+C_{1},
$$

and since $A(b)=0$, we have to

$$
\begin{aligned}
A(t) & =-\int_{a}^{t} \frac{x_{2}(\varepsilon)}{K} f(\varepsilon) \frac{1}{\varepsilon^{1-\alpha}} d \varepsilon \\
& +\int_{a}^{b} \frac{x_{2}(\varepsilon)}{K} f(\varepsilon) \frac{1}{\varepsilon^{1-\alpha}} d \varepsilon=\int_{t}^{b} \frac{x_{2}(\varepsilon)}{K} f(\varepsilon) \frac{1}{\varepsilon^{1-\alpha}} d \varepsilon .
\end{aligned}
$$

Analogously

$$
B(t)=-\int_{a}^{t} \frac{x_{1}(\varepsilon)}{K} f(\varepsilon) \frac{1}{\varepsilon^{1-\alpha}} d \varepsilon .
$$

Thus, we obtain

$$
\begin{aligned}
x(t) & =A(t) x_{1}(t)+B(t) x_{2}(t) \\
& =\int_{t}^{b} \frac{x_{1}(t) X_{2}(\varepsilon)}{K} f(\varepsilon) \frac{1}{\varepsilon^{1-\alpha}} d \varepsilon \\
& +\int_{a}^{t} \frac{X_{1}(\varepsilon) x_{2}(t)}{K} f(\varepsilon) \frac{1}{\varepsilon^{1-\alpha}} d \varepsilon \\
& =\int_{a}^{b} G_{3}^{\alpha}(t, \varepsilon) f(\varepsilon) \frac{1}{\varepsilon^{1-\alpha}} d \varepsilon,
\end{aligned}
$$

where we have the following

$$
G^{\alpha}(t, \varepsilon)=\left\{\begin{array}{cc}
\frac{1}{K} x_{1}(t) x_{2}(\varepsilon) & a \leq t<\varepsilon \\
\frac{1}{K} x_{1}(\varepsilon) x_{2}(t) & \varepsilon \leq t<b
\end{array} .\right.
$$

which is the Green's Function.

Example 2. By using the Green's Function, we want to solve the following system

$$
\left.\begin{array}{rl}
T_{\alpha} T_{\alpha} x(t)+x(t)=e^{t^{\alpha} / \alpha} \quad t \in\left[0,(\alpha \pi)^{1 / \alpha}\right] \\
x(0)=0 \\
T_{\alpha} x\left((\alpha \pi)^{1 / \alpha}\right)=0
\end{array}\right\} .
$$

First, we find the conformable Green's Function of the homogeneous system.

We have following:

$$
\begin{aligned}
& x(t)=A \cos \left(\frac{t^{\alpha}}{\alpha}\right)+B \sin \left(\frac{t^{\alpha}}{\alpha}\right) \\
& X(0)=0=A \\
& T_{\alpha} x\left((\alpha \pi)^{1 / \alpha}\right)=0=B .
\end{aligned}
$$

Therefore, the conformable Green's Function exists. This function can be written as

$$
G^{\alpha}(t, \varepsilon)=\left\{\begin{array}{cc}
\cos \left(\frac{\varepsilon^{\alpha}}{\alpha}\right) \sin \left(\frac{t^{\alpha}}{\alpha}\right) & 0 \leq t<\varepsilon \\
\sin \left(\frac{\varepsilon^{\alpha}}{\alpha}\right) \cos \left(\frac{t^{\alpha}}{\alpha}\right) \quad \varepsilon<t \leq(\alpha \pi)^{1 / \alpha}
\end{array} .\right.
$$

Therefore, our intended solution can be written as follows:

$$
\begin{aligned}
x(t)=-\int_{0}^{(\alpha \pi)^{1 / \alpha}} G^{\alpha}(t, \varepsilon) e^{\varepsilon^{\alpha} / \alpha} \frac{1}{\varepsilon^{1-\alpha}} d \varepsilon \\
=-\int_{0}^{t} \sin \left(\frac{\varepsilon^{\alpha}}{\alpha}\right) \cos \left(\frac{t^{\alpha}}{\alpha}\right) e^{\varepsilon^{\alpha} / \alpha} \frac{1}{\varepsilon^{1-\alpha}} d \varepsilon \\
-\int_{t}^{(\alpha \pi)^{1 / \alpha}} \cos \left(\frac{\varepsilon^{\alpha}}{\alpha}\right) \sin \left(\frac{t^{\alpha}}{\alpha}\right) e^{\varepsilon^{\alpha} / \alpha}(\alpha \pi)^{1 / \alpha} d \varepsilon \\
=-\left.\frac{1}{2} \cos \left(\frac{t^{\alpha}}{\alpha}\right)\left(e^{\varepsilon^{\alpha} / \alpha}\left[\sin \left\{\frac{\varepsilon^{\alpha}}{\alpha}\right\}-\cos \left\{\frac{\varepsilon^{\alpha}}{\alpha}\right\}\right]\right)\right|_{\varepsilon=0} ^{\varepsilon=t} \\
-\left.\frac{1}{2} \sin \left(\frac{t^{\alpha}}{\alpha}\right)\left(e^{\varepsilon^{\alpha} / \alpha}\left[\sin \left\{\frac{\varepsilon^{\alpha}}{\alpha}\right\}-\cos \left\{\frac{\varepsilon^{\alpha}}{\alpha}\right\}\right]\right)\right|_{\varepsilon=t} ^{\varepsilon=(\alpha \pi)^{1 / \alpha}} \\
=-\frac{1}{2} \cos \left(\frac{t^{\alpha}}{\alpha}\right)\left(e^{\varepsilon^{\alpha} / \alpha}\left[\sin \left\{\frac{t^{\alpha}}{\alpha}\right\}-\cos \left\{\frac{t^{\alpha}}{\alpha}\right\}+1\right]\right) \\
-\frac{1}{2} \sin \left(\frac{t^{\alpha}}{\alpha}\right)\left(e^{\pi}-e^{t^{\alpha} / \alpha}\left[\sin \left\{\frac{t^{\alpha}}{\alpha}\right\}-\cos \left\{\frac{t^{\alpha}}{\alpha}\right\}\right]\right) \\
\left.e^{\pi}\left(\frac{t^{\alpha}}{\alpha}\right)-\frac{t^{\alpha}}{2} e^{\pi} \sin \left(\frac{t^{\alpha}}{\alpha}\right) \cdot \cos \frac{1}{2}\right) \\
=
\end{aligned}
$$

Finally, we investigate the generalized Hyers-Ulam stability of the conformable linear inhomogeneous differential equation of order $\alpha+\alpha$ (21) in the class of continuously twice $\alpha$-differentiable functions.

Theorem 19. Let $p, q, f:[a, b] \rightarrow R$ be continuous functions and let $p$ be $\alpha$-differentiable function on $[a, b]$. Assume that the conformable homogeneous differential equation (20) 
has its only solution as the identically null function. If a twice continuously $\alpha$-differentiable function $x:[a, b] \rightarrow R$ satisfies the inequality

$$
\left|T_{\alpha}\left(p(t) T_{\alpha} x(t)\right)-q(t) x(t)+f(t)\right| \leq \varphi(t),
$$

for all $t \in[a, b]$, where $\varphi:[a, b] \rightarrow[0, \infty)$ is given that such of the following integrals exists, then there exists a solution $x_{0}:[a, b] \rightarrow R$ of $(21)$ such that

$$
\begin{aligned}
\left|x(t)-x_{0}(t)\right| & \leq \frac{1}{|K|}\left(\left|x_{1}(t)\right| \int_{b}^{t}\left|x_{2}(\varepsilon)\right| \varphi(\varepsilon) \frac{1}{\varepsilon^{1-\alpha}} d \varepsilon\right. \\
& \left.+\left|x_{2}(t)\right| \int_{a}^{t}\left|x_{1}(\varepsilon)\right| \varphi(\varepsilon) \frac{1}{\varepsilon^{1-\alpha}} d \varepsilon\right),
\end{aligned}
$$

where $K$ is a nonzero constant and $x_{1}(t)$ and $x_{2}(t)$ are two linearly independent solutions of (20a) that verify (20b) and (20c), respectively (see Theorem 18).

Proof. If we define a continuous function $s:[a, b] \rightarrow R$ by

$$
s(t)=T_{\alpha}\left(p(t) T_{\alpha} x(t)-q(t) x(t)\right),
$$

for all $t \in[a, b]$, then it follows (22) that

$$
|s(t)+f(t)| \leq \varphi(t),
$$

for all $t \in[a, b]$. In view of Theorem 18 and (24), we have

$$
\begin{aligned}
x(t) & =-\int_{a}^{b} G^{\alpha}(t, \varepsilon) s(\varepsilon) \frac{1}{\varepsilon^{1-\alpha}} d \varepsilon \\
& =-\int_{t}^{b} \frac{x_{1}(t) x_{2}(\varepsilon)}{K} s(\varepsilon) \frac{1}{\varepsilon^{1-\alpha}} d \varepsilon \\
& -\int_{a}^{t} \frac{x_{1}(\varepsilon) x_{2}(t)}{K} s(\varepsilon) \frac{1}{\varepsilon^{1-\alpha}} d \varepsilon
\end{aligned}
$$

where $K$ is a nonzero constant because $x_{1}(t)$ and $x_{2}(t)$ are two linearly independent solutions of (20a) that verify (20b) and (20c), respectively (see Theorem 18). We now define a function $x_{0}:[a, b] \rightarrow R$ by

$$
\begin{aligned}
x_{0}(t) & =\int_{t}^{b} \frac{x_{1}(t) x_{2}(\varepsilon)}{K} f(\varepsilon) \frac{1}{\varepsilon^{1-\alpha}} d \varepsilon \\
& +\int_{a}^{t} \frac{x_{1}(\varepsilon) x_{2}(t)}{K} f(\varepsilon) \frac{1}{\varepsilon^{1-\alpha}} d \varepsilon,
\end{aligned}
$$

for all $t \in[a, b]$. According to Theorem 18, it is obvious that $x_{0}$ is a solution of (21). Moreover, it follows from (25), (26) and (27) that

$$
\begin{aligned}
\left|x(t)-x_{0}(t)\right| \leq \mid-\int_{t}^{b} \frac{x_{1}(t) x_{2}(\varepsilon)}{K}(s(\varepsilon)+f(\varepsilon)) \frac{1}{\varepsilon^{1-\alpha}} d \varepsilon \\
-\int_{a}^{t} \frac{x_{1}(\varepsilon) x_{2}(t)}{K}(s(\varepsilon)+f(\varepsilon)) \frac{1}{\varepsilon^{1-\alpha}} d \varepsilon \mid \\
\leq \frac{1}{|K|}\left(\left|x_{1}(t)\right| \int_{b}^{t}\left|x_{2}(\varepsilon)\right| \varphi(\varepsilon) \frac{1}{\varepsilon^{1-\alpha}} d \varepsilon\right. \\
\left.+\left|x_{2}(t)\right| \int_{a}^{t}\left|x_{1}(\varepsilon)\right| \varphi(\varepsilon) \frac{1}{\varepsilon^{1-\alpha}} d \varepsilon\right)
\end{aligned}
$$

for all $t \in[a, b]$.

Remark 4. Theorem 19 reduces to [22] (Theorem 3.2) in the case $\alpha=0$ and using the Green's Function.

\section{Conclusion}

In this research paper, we have proposed some results referring to the conformable boundary value problems. The conformable Sturm-Picone identity of order $\alpha+\alpha$ has been proven, and its Sturm's theorems of comparison and separation have been successfully established. As in the classical case, an important application of the Sturm's comparison theorem is to provide a clear understanding of the zero set of non-trivial solutions of the conformable Bessel's equation. For a conformable Sturm-Liouville system, we have defined the Green's function and established its properties. The conformable Green's function is applied to construct the solution of the inhomogeneous problem of Sturm-Liouville, whose associated homogeneous problem has its only solution as the identically null function. Finally, we have proved the generalized Hyers-Ulam stability of the conformable linear inhomogeneous differential equation of order $\alpha+\alpha$ (21) in the class of continuously twice $\alpha$-differentiable functions.

\section{Acknowledgments}

All authors would like to express their very great appreciation to all referees and editorial board members for their helpful suggestions and valuable comments.

\section{Conflict of interest}

All authors declare no conflicts of interest in this research paper. 
1. A. A. Kilbas, H. M. Srivastava, and J. J. Trujillo, Theory and Applications of Fractional Differential Equations, 1st ed. (Elsevier, Amsterdam, 2006).

2. K. S. Miller and B. Ross, An Introduction to the Fractional Calculus and Fractional Differential Equations (Wiley, New York, 1993).

3. R. Khalil, M. Al Horani, A. Yousef, and M. Sababheh, A new definition of fractional derivative, J. Comput. Appl. Math. 264 (2014) 65, https://doi.org/10.1016/j.cam. 2014.01 .002

4. T. Abdeljawad, On conformable fractional calculus, J. Comput. Appl. Math. 279 (2015) 57, https://doi.org/10. $1016 /$ j.cam.2014.10.016.

5. O. S. Iyiola and E. R. Nwaeze, Some New Results on the New Conformable Fractional Calculus with Applicaation Using D'Alambert Approach, Prog. Fract. Differ. Appl. 2 (2016) 115, https://doi.org/10.18576/pfda/020204

6. A. Atangana, D. Baleanu, and A. Alsaedi, New properties of conformable derivative, Open Math 13 (2015) 889, https : //doi.org/10.1515/math-2015-0081

7. N. Y. Gözütok and U. Gözütok, Multi-variable conformable fractional calculus, Filomat 32 (2018) 45, https://doi. org/10.2298/FIL1801045G

8. F. Martínez, I. Martńez, and S. Paredes, Conformable Euler's theorem on homogeneous functions, Comput. Math. Methods 1 (2019) e1048, https://doi.org/10.1002/cmm4. 1048

9. E. Ünal, A. Gökdogan, and E. Çelik, Solutions of Sequential Conformable Fractional Differential Equations around an Ordinary Point and Conformable Fractional Hermite Differential Equation, Br. J. Appl. Sci. Technol. 10 (2015) 18590, https://doi.org/10.9734/BJAST/2015/18590

10. M. Al Masalmeh, Series method to solve conformable fractional Riccati differential equations, Int. J. Appl. Math. Res. 6 (2017) 30, https://doi.org/10.14419/ijamr. v6i1.7238.

11. M. Al Horani and R. Khalil, Total fractional differentials with applications to exact fractional differential equations, Int. J. Comput. Math. 95 (2018) 1444, https://doi.org/10. $1080 / 00207160.2018 .1438602$

12. M. Al Horani, M. Abu Hammad, and R. Khalil, Variation of parameters for local fractional nonhomogenous linear differential equations, J. Math. Comput. Sci. 16 (2016) 147, https: //doi.org/10.22436/jmcs.016.02.03

13. R. Khalil, M. Al Horani, and D. Anderson, Undetermined coefficients for local fractional differential equations, J. Math. Comput. Sci. 16 (2016) 140, https://doi.org/10.22436/ jmcs.016.02.02

14. M. Abu Hammad and R. Khalil, Abel's formula and Wronskian for conformable fractional differential equations, Int. J. Differ. Equ. Appl. 13 (2014) 177.

15. R. Khalil and M. Abu Hammad, Legendre fractional differential equation and Legendre fractional polynomials, Int. J. Appl. Math. Res. 3 (2014) 214, https : / / doi .org/10.14419/ i jamr.v3i3.2747
16. F. S. Silva, D. M. Moreira, and M. A. Moret, Conformable Laplace Transform of Fractional Differential Equations, Axioms 7 (2018) 55, https : / / doi .org/10.3390/ axioms 7030055

17. Z. Al-Zhour, N. Al-Mutairi, F. Alrawajeh, and R. Alkhasawneh, Series solutions for the Laguerre and Lane-Emden fractional differential equations in the sense of conformable fractional derivative, Alexa. Eng. J. 58 (2019) 1413, https://doi. org/10.1016/j.aej.2019.11.012

18. M. Abu Hammad, H. Alzaareer, H. Al-Zoubi, and H. Dutta, Fractional Gauss hypergeometric differential equation, J. Interscip. Math. 22 (2019) 1113, https://doi.org/10. $1080 / 09720502.2019 .1706838$

19. W. R. Derrick and S. I. Grossman, Elementary Differential Equations with Applications (Addison-Wesley, Reading, 1981).

20. C. T. Fulton, L. Wu, and S. Pruess, A Sturm separation theorem for a linear 2 nth order self-adjoint differential equation, Int. J. Stoch. Anal. 8 (1995) 464071, https://doi.org/ $10.1155 /$ S1048953395000037

21. M. Kaabar, Novel Methods for Solving the Conformable Wave Equation, J. New Theor. 31 (2019) 56.

22. G. Choi and S.-M. Jung, Invariance of Hyers-Ulam stability of linear differential equations and its applications, Adv. Differ. Equ. 2015 (2015) 277, https : / doi.org/10.1186/ s13662-015-0617-1

23. F. Martínez, I. Martńez, M. K. A. Kaabar, R. Ortíz-Munuera, and S. Paredes, Note on the Conformable Fractional Derivatives and Integrals of Complex-valued Functions of a Real Variable, IAENG Int. J. Appl. Math. 50 (2020) 609.

24. F. Martínez, I. Martínez, M. K. A. Kaabar, and S. Paredes, New results on complex conformable integral, AIMS Math. 5 (2020) 7695, https://doi.org/10.3934/math.2020492

25. P. M. Guzmán, G. Langton, L. M. Lugo Motta Bittencurt, J. Medina, and J. E. Nápoles Valdás, A new definition of a fractional derivative of local type, J. Math. Anal. 9 (2018) 88.

26. J. E. Nápoles Valdés, P. M. Guzmán, and L. M. Lugo, Some New Results on Nonconformable Fractional Calculus, $A d v$. Dyn. Syst. Appl. 13 (2018) 167.

27. A. Fleitas, J. A. Méndez-Bermúdez, J. E. Nápoles Valdés, and J. M. Sigarreta Almira, On fractional Liénard-type systems, Rev. Mex. Fis. 65 (2019) 618, https: //doi .org/10.31349/ RevMexFis.65.618

28. P. M. Guzmán and J. E. Nápoles Valdés, A note on the oscillatory character of some non conformable generalized Liénard system, Adv. Math. Models Appl. 4 (2019) 127.

29. A. Fleitas, J. F. Gómez-Aguilar, J. E. Nápoles Valdés, J. M. Rodríguez, and J. M. Sigarreta, Analysis of the local Drude model involving the generalized fractional derivative, $O p$ tik 193 (2019) 163008, https://doi.org/10.1016/j. ijleo.2019.163008

30. J. E. Nápoles Valdés, J. M. Rodríguez, and J. M. Sigarreta, New Hermite- Hadamard Type Inequalities Involving NonConformable Integral Operators, Symmetry 11 (2019) 1108, https://doi.org/10.3390/sym11091108

31. F. Martínez, P. O. Mohammed, and J. E. Nápoles Valdés, Nonconformable fractional Laplace transform, Kragujevav J. Math. 46 (2022) 341 (to be published). 
32. R. Abreu-Blaya et al., On the conformable fractional logistic models, Math. Methods Appl. Sci. 43 (2020) 4156, https: //doi.org/10.1002/mma.6180

33. J. E. Nápoles Valdés and C. Tunç, On the boundedness and oscillation of non-conformable Liénard equation, J. Fract. Calc. Appl. 11 (2020) 92.

34. J. E. Nápoles Valdés, P. M. Guzmán, L. M. Lugo, and A. Kashuri, The local generalized derivative and Mittag-Leffler function, Sigma J. Eng. Nat. Sci. 38 (2020) 1007.

35. J. E. Nápoles Valdés and M. N. Quevedo Cubillos, On the Oscillatory Nature of Some Generalized Emden-Fowler Equation, Punjab Univ. J. Math. 52 (2020) 97.

36. F. Martínez and J. E. Nápoles Valdés, Towards a Nonconformable Fractional Calculus of n-Variables, J. Math. Appl. 43 (2020) 87, https://doi.org/10.7862/rf.2020. 6
37. P. M. Guzmán, L. M. Lugo Motta Bittencurt, and J. E. Nápoles Valdés, On the stability of solutions of fractional non conformable differential equations, Stud. Univ. Babes-Bolyai Math. 65 (2020) 495.

38. J. E. Nápoles Valdés, M. N. Quevedo Cubillos, and A. R. Gómez Plata, On the asymptotic behavior of a generalized nonlinear equation, Sigma J. Eng. Nat. Sci. 38 (2020) 2109.

39. J. E. Nápoles Valdés, P. M. Guzmán, and L. M. Lugo Motta Bittencurt, A note on the qualitative behavior of some nonlinear local improper conformable differential equations, J. Fract. Calc. Nonlinear Syst. 1 (2020) 13, https : / / doi.org/10. $48185 / j$ fens.v1i1.48

40. F. Martínez, I. Martínez, M. K. A. Kaabar, and S. Paredes, Some New Results on Conformable Fractional Power Series, Asia Pac. J. Math. 7 (2020) 31, https://doi.org/10. 28924/AP JM/7-31 